\title{
A Novel Particle Failure Criterion for Cleavage Fracture Modelling Allowing Measured Brittle
} Particle Distributions

P. M. James ${ }^{a, *}$, M. Ford ${ }^{a}$ and A.P. Jivkov ${ }^{b}$

a - AMEC Clean Energy, Walton House, Birchwood Park, Risley, Warrington, Cheshire, UK, WA3 6GA b - School of Mechanical, Aerospace and Civil Engineering, The University of Manchester, Manchester M13 9PL, UK

* Corresponding Author, Tel: +44(0)1925 253901; Fax: +44(0)1925 252285; E-mail: peter.james2@amec.com (P. James). Keywords:

Local Approach Modelling; Toughness Shift; Irradiation Embrittlement; RPV steel; Local Failure

Criterion; Transition Temperature Regime

\begin{abstract}
Most of the existing local approaches for cleavage fracture derive from the assumptions that global failure is a weakest-link event and that only the tail of the size distribution of micro-crack initiating features is significant. This appears to be sufficient in predicting lower shelf toughness under high constraint conditions, but may fail when attempting to predict toughness in the transition region or for low constraint conditions when using the same parameters. While coupled ductile damage models with Beremin-like failure probability could be useful in the transition region, uncoupled models with "a posteriori" probability calculations are advantageous to the engineering community. Cleavage toughness predictions in the transition regime, which can be extended to low constraint conditions, are here made with improved criterion for particle failure and experimentally based size distribution of initiators for specific RPV steel. The model is shown to predict experimentally measured locations of cleavage initiators. Further, the model predicts the fracture toughness in a large part of the transition region and accurately predicts the measured shift with irradiation, although further works is required to improve the model's predictions at higher levels of fracture toughness and for low constraint conditions, which are expected to be achieved by a better accounting for plasticity. All results are obtained without changes in model parameters. This suggests that the model can be used as a tool for analysing toughness changes due to constraint and temperature driven plasticity changes.
\end{abstract}

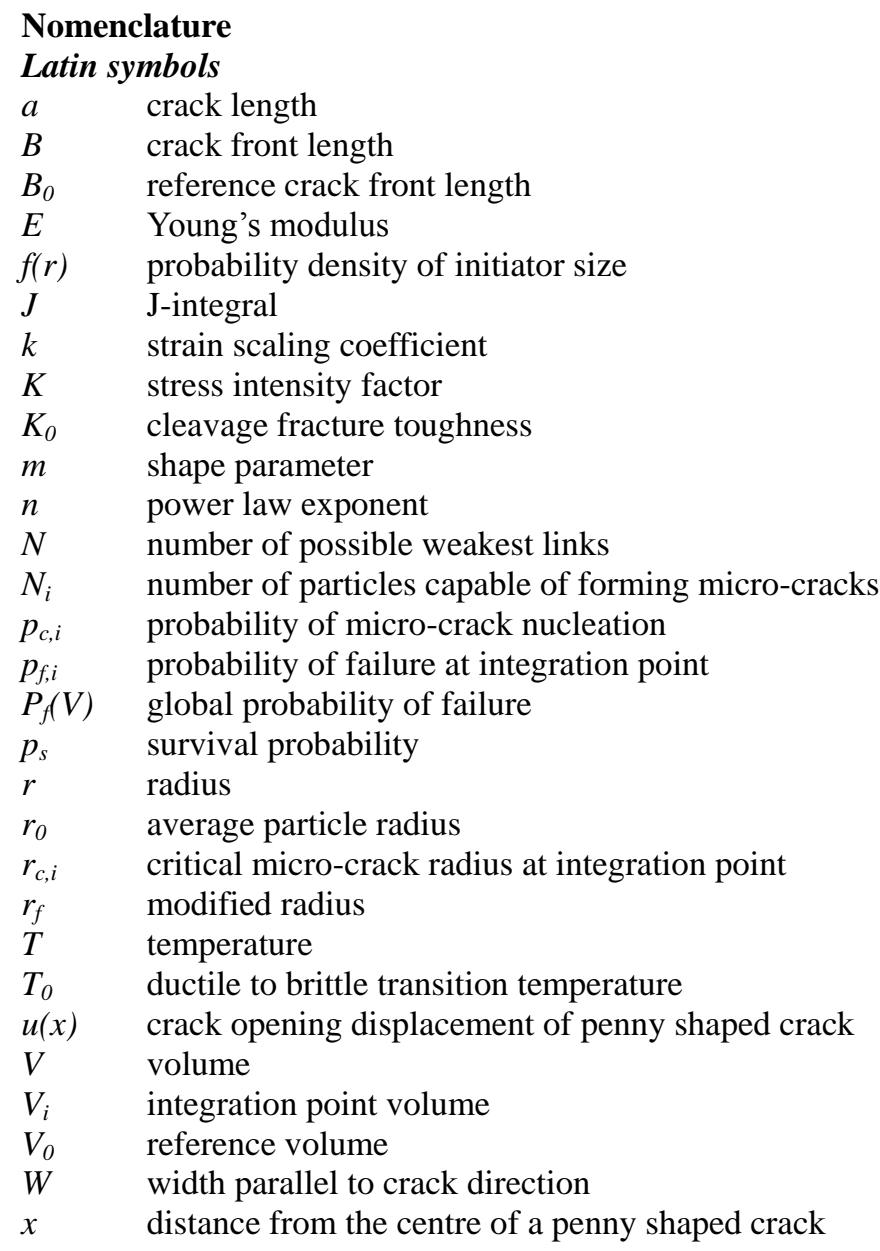




\title{
Greek symbols
}

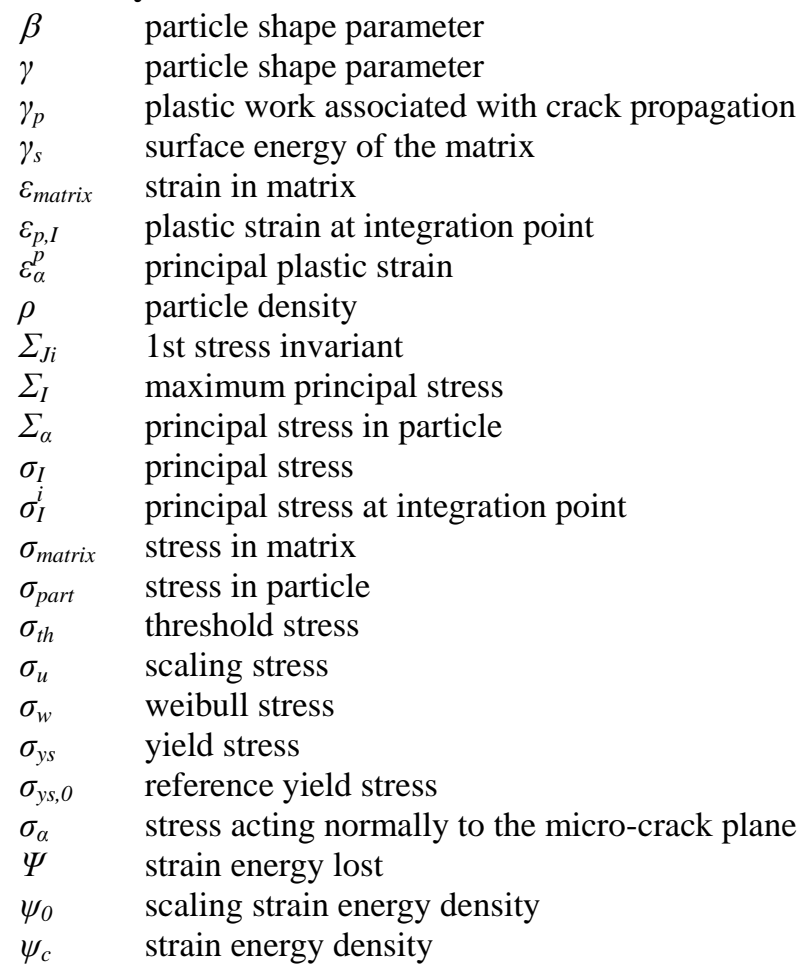

\author{
Abbreviations \\ CTOD crack tip opening displacement \\ DBT ductile to brittle transition \\ FE finite element \\ LA local approaches \\ PCCV pre-cracked charpy V notch specimen \\ RPV reactor pressure vessel \\ SEN(B) single edged notch bend test specimen
}

\section{Introduction}

Safety assessment and life extension decisions in nuclear plant require reliable methodologies for predicting changes in cleavage fracture toughness behaviour of ferritic reactor pressure vessel (RPV) steels due to irradiation and defect geometry effects. Local approaches (LA) to cleavage fracture are promising as these could account for the micro-mechanisms involved in the cleavage failure phenomenon, such as the nucleation of micro-cracks at second-phase brittle particles, the propagation of such micro-cracks within grains and the propagation of a critical micro-crack leading to component failure [1]. The pioneering LA to cleavage, proposed by the Beremin group [2], is based on two main assumptions: that the global failure probability is a weakest-link event and that the individual failure probabilities are dictated by local mechanical fields and specific microstructure data such as the size distribution and number density of cleavage-initiating particles. Assuming that the tail of the size distribution can be approximated by a power-law, the weakest-link statistics leads to a global failure probability expressed as a Weibull-type function of a generalised stress, the Weibull stress $\sigma_{w}[2,3]$ :

$$
P_{f}(V)=1-\exp \left[-\left(\frac{\sigma_{w}}{\sigma_{u}}\right)^{m}\right]
$$

The shape parameter, $m$, and scaling stress, $\sigma_{u}$, of the Weibull function can be calibrated using fracture toughness data obtained with a particular crack geometry at a given temperature and corresponding finite element (FE) analysis [4]. For calibrations performed with geometries with high 
constraint at the crack tip at or below the ductile-to-brittle transition temperature, $T_{0}$, which defines the temperature at which the 63.2 percent cleavage fracture toughness, $K_{O}=108 \mathrm{MPa} \sqrt{\mathrm{m}}$, the shape and scale parameters obtained can be used with sufficiently good accuracy to predict cleavage fracture toughness of other high-constraint geometries at temperatures below $T_{0}$ [2-4]. However, attempts to use the same parameters to calculate cleavage fracture toughness under lower constraint conditions or at higher temperatures leads to predictions that do not match experimental values. For cleavage fracture toughness data obtained at a given temperature with low and high constraint geometries, independent calibrations would typically show that the low-constraint $m$ is smaller than the high-constraint $m$ when $\sigma_{u}$ is constant, or the low constraint $\sigma_{u}$ is higher than the high constraint $\sigma_{u}$, where $m$ is constant, see e.g. [5]. This suggests that the increase in plastic deformation introduced by the lower constraint requires a reduction of $m$ to make reliable predictions. The methodology proposed in [5] for cross-calibration of $m$ between high and low constraint geometries has shown promise [5, 6], but remains limited to temperatures well below $T_{0}$, where the lower constraint conditions do not introduce a substantial increase in plastic deformations relative to the high constraint case. This is problematic when used to predict cleavage fracture toughness for short cracks in irradiated material, where the $T_{0}$ temperature is shifted to higher values. To predict the irradiation effects on cleavage one needs a reliable model for the ductile-to-brittle transition (DBT) regime. Here, however, the Weibull parameters need to be varied to match experimental data which allows two options. One possibility is to keep the shape parameter constant, which leads to temperature dependence of the scale parameter, $\sigma_{u}[7,8]$. Another possibility is to keep the scale parameter constant, which leads to temperature dependence of the shape parameter, $m[9,10]$. For the low-constraint situation, $m$ needs to be reduced with increasing temperature, i.e. with enhanced plasticity.

Considering the physical basis for the Beremin LA [2, 3], the current state of affairs is not satisfactory, because the Weibull parameters must depend exclusively on the material microstructure. If the model for individual failure probability accounted adequately for the local fields and microstructure effects on particle failure, and global failure was a weakest-link event, then the changes in plasticity due to changes in constraint or temperature should be already accounted for, leaving the Weibull parameters constants. In particular, $m$ should be linked to the shape of size distribution of cleavage initiation particles, while $\sigma_{u}$ should depend on the elastic properties and surface energy of the material, in addition to the scale of the particle size distribution. Since these parameters do not change noticeably with constraint or temperature, the need to vary $m$ and/or $\sigma_{u}$ to accurately predict the fracture toughness as plastic deformation increases suggests that the link between physics and mechanics does not adequately capture the effects of high levels of plasticity. One possibility is that the individual failure probability model does not account adequately for the mechanical and particle size effects. A second possibility is that, with the increase of plastic deformation, the population of microcracks that need to be accounted for in the weakest-link statistics becomes larger than the tail of the distribution, approximated by power-law in the Weibull-stress models. A third possibility is that the weakest-link assumption becomes increasingly invalid with increasing plasticity, and micro-crack interaction effects need to be accounted for.

To improve the individual failure probability, local plastic strains has been incorporated in the calculation of $\sigma_{w}$ in modifications of the Beremin model [11, 12] as well as in incremental formulations [13]. Recently, in addition to plastic strains the local stress triaxiality has also been incorporated [14] into $\sigma_{w}$. In a previous work [15], these models have been compared with the original Beremin model and demonstrated that they provide improvements in the predicted failure probability profiles ahead of cracks with different levels of constraint. This comparison has been done against a large set of experimental data for the locations of cleavage initiation sites reported in [14]. However, the prediction of the cleavage fracture toughness temperature dependence with the Weibull based models remained unsatisfactory when performed with single set of Weibull parameters calibrated at $T_{0}$. The effect of particle size on local failure probability was first proposed in [16]. The model now known as WST, however, remained as a microstructure-informed local model and to the authors' knowledge has not previously been applied for global failure predictions. A different direction of work considers ductile damage prior to cleavage in the DBT regime, employing damage models for the material behaviour coupled with subsequent calculation of failure probability following Beremin [17, 18]. This approach has the potential to better capture the fracture behaviour in the DBT, but the models are presently too simplified with no interaction of void growth and micro-cracking at the microstructural level [19]. Until advanced coupled models are developed and validated, the wider 
engineering community would benefit from a simpler uncoupled cleavage fracture model for the DBT region which is able to predict fracture toughness through the DBT region and take into account changes in material due to irradiation and changes in crack tip constraint. One direction of work on uncoupled modelling, known as the Prometey model, has been in improving the local failure criterion by taking into account the probability of particle rupture and micro-crack propagation [20]. The effect of particle size, however, still needs to be accounted for in this model.

In [15] a model incorporating mechanical and particle size effects was initially considered and it demonstrated that it provides improved predictions for failure probability profiles. In this work application of further developments to this model are applied to predict cleavage fracture toughness in the DBT regime. The focus of the model development was on the first two possibilities mentioned above: to improve individual probability of failure and to account directly for the real particle size distribution rather than approximating the tail with a power law relation. However, the weakest-link assumption for the calculation of global probability is maintained. The proposed model, as the discussed local approaches, is applicable to macroscopically homogeneous materials.

It is noted that the Modified Beremin, Bordet and WST model can incorporate the effects of constraint and irradiation through the variation of explicit terms. The goal of our development is to have a model where no terms require variation. Our investigation calibrates the different approaches at a single temperature in the un-irradiated condition with high constraint and evaluates how the approaches perform when used predictively, i.e. can the change in fracture toughness due to irradiation and changes in constraint be predicted using only the changes in tensile properties and crack geometry.

\section{Theory and model}

\subsection{Statistical Basis}

LA methods share a common philosophy based on two distinct components. Firstly, the local mechanical fields, defined assuming a macroscopically homogenous material, provide a local or 'individual' probability of failure when linked to the size distribution of the micro-crack initiators. The individual probability of failure at location $i$ is

$$
p_{f, i}=\int_{r_{c, i}}^{\infty} p_{c, i} f(r) d r
$$

where $f(r)$ is the probability density of initiators' sizes, $p_{c, i}$ is the probability of micro-crack nucleation (and propagation, assuming weakest link), and $r_{c, i}$ is the critical micro-crack size at location $i$. Note that $p_{c} f(r)$ is the probability density of nucleated micro-crack sizes. Existing LAs can be recast into Eq. (2) albeit with different definitions of $p_{c}$ and $r_{c}$. For example in [2-4] $p_{c}=0$ or 1 for zero and non-zero plastic strains. In $[11,12] p_{c}$ scales with the equivalent plastic strain, while in [14] $p_{c}$ scales with the equivalent plastic strain and exponent of the stress triaxiality. In [13] $p_{c}$ is a more complex function of stress and plastic strain increments. In all cases $r_{c}$ is defined via Griffith or plasticity-modified Griffith criterion. Common feature of $[2-4,11-14]$ is that $p_{c}$ is independent of particle size.

Secondly, it is assumed that the individual failure events are independent. This allows the weakestlink argument to be invoked for calculating the global failure probability, so that

$$
P_{f}(V)=1-\prod_{i=1}^{N}\left(1-p_{f, i}\right)
$$

where $N$ is the number of possible weakest-links, i.e. potential active micro-cracks, in volume $V$. In practice, LAs are applied to FE solutions of cracked components, where the mechanical fields are constant within an integration point volume, $V_{i}$. The failure probability of such a volume is thus

$$
P_{f}\left(V_{i}\right)=1-\left(1-p_{f, i}\right)^{N_{i}}=1-\left(1-p_{f, i}\right)^{\rho_{i} V_{i}}
$$

where $N_{i}=\rho_{i} V_{i}$ is the number of particles capable of failing and forming micro-cracks in $V_{i}$, and 
$\rho_{i}$ is the density of these particles. Strictly speaking $\rho_{i}=\rho p_{c, i}$, where $\rho$ is the density of initiating particles in the material. The component probability of failure is then calculated by repeated application of Eq. (4) to get

$$
P_{f}(V)=1-\prod_{i=1}^{I P}\left(1-p_{f, i}\right)^{\rho_{i} V_{i}}
$$

where the product is taken over all integration points. When $p_{c}$ is independent of particle size, Eq. 2 can have a closed-form solution in terms of critical micro-crack sizes, $r_{c}$. With a power law approximation for particle sizes and assuming small individual failure probabilities, Eq. (5) yields the Weibull-stress function in Eq. (1). Here Eq. (5) is used directly to calculate the global failure probability, because the experimental particle size distribution, given in Section 2.2, and a new expression for individual failure probability, derived in Section 2.3, do not allow for closed form solution of Eq. (2) and hence a corresponding definition of a generalised stress.

\subsection{Existing Local Approach Models}

\subsubsection{Original Beremin Model}

The Beremin Model forms the basis of the conventional approach to the prediction of cleavage fracture within the local approach construct. The basic premise of the original Beremin Model [2] is that the probability of probability of rupture is directly linked to the maximum principal stress and the failure event follows the weakest link principle, where the formation of a single micro-crack ruptures the surrounding matrix and leads to global failure. A measure of the uncertainty in the failure (i.e. that linked to the particle size distribution, $m$, typically a second phase carbide) is adopted in the calculation. If the principal stress is seen to go significantly beyond the provided value of the reference stress, $\sigma_{u}$, (which is calibrated) the probability of rupture is seen to rapidly approach unity from Eqs. (6) and (7),

$$
\begin{aligned}
& P_{f}=1-\exp \left[-\left(\sigma_{w} / \sigma_{u}\right)^{m}\right] \\
& \sigma_{w}=\sqrt[m]{\sum_{i} \sigma_{I}^{i^{m}} \frac{V_{i}}{V_{O}}}
\end{aligned}
$$

where $\sigma_{I}$ is the principal stress, $V_{i}$ is the volume being considered and $V_{0}$ is the reference volume. Where values are denoted by $i$ this is the value associated with a finite volume, such as that in a finite element. The only variables available for calibration are $\sigma_{u}, m, V_{0}$. Note that the form of

Eq. (7) means that $\sigma_{u}, m$ and $V_{0}$ are related as $\sigma_{u} V_{0} \frac{1}{m}=$ const. Once calibrated for a given geometry and irradiation condition the Weibull parameters can be transferred to another to calculate the probability of cleavage fracture.

However, some difficulties have been observed during the use of the Beremin Model, which are [4]: (1) Different shape factors $m$ are obtained for the same material depending on crack geometry (i.e. constraint), temperature or loading rate. (2) The iterative calibration procedure for the shape parameter $m$ diverges or produces very high $m$ values, which do not relate well with the recorded scatter in experimental data. (3) The model sometimes gives poor cleavage fracture predictions.

\subsubsection{Modified Beremin Model}

The Modified Beremin Model [12] provides a further approach to the prediction of cleavage fracture within a local approach construct, and is based on the original Beremin Model. The modification is the replacement of the Weibull stress $\sigma_{w}$ calculation in Eq. (7) with that defined by Eq. (8),

$$
\sigma_{w}=\sqrt[m]{\sum_{i}{\sigma_{I}^{i}}^{m} \exp \left(-\frac{m \varepsilon_{p, I}}{k}\right) \frac{V_{i}}{V_{0}}}
$$


which includes the principal plastic strains, $\varepsilon_{p, I}$, and scaling coefficient $k$, which can be used as a calibration parameter. The addition of the strain term allows for the Modified Beremin Model to better take into account the effect of constraint, improving the ability to apply the model to geometries other than the calibration geometry. This can also be adopted with the constraints that the probability of failure is increasing with a threshold condition when applied to warm pre stress conditions.

\subsubsection{Bordet Model}

The Bordet Model [13] is a modification of the original Beremin Model with the aim of addressing some of the difficulties that have appeared in the latter's use. The modification is the replacement of the Weibull stress $\sigma_{w}$ calculation in Eq. 6 with that defined by Eq. 9,

$$
\sigma_{w}=\sqrt[m]{\left(\sum_{i} \frac{\sigma_{y s}\left(T, \dot{\varepsilon}_{p}\right)}{\sigma_{y s, 0}}\left(\sigma_{I}^{i}\left(\varepsilon_{p}^{i}, d V\right)-\sigma_{t h}^{m}\right) d \varepsilon_{p}^{i}\right) \frac{V_{i}}{V_{O}}}
$$

where $\sigma_{y s}\left(T, \dot{\varepsilon}_{p}\right)$ is the yield stress at the temperature and strain rate at the integration point, $\sigma_{y s, 0}$ is a reference yield stress at a reference temperature, which has been taken as equal to the $T_{0}$ temperature, $\sigma_{t h}$ is a threshold stress, $d \varepsilon_{p}^{i}$ is the change in strain. The term $\sigma_{I}^{i}{ }^{m}\left(\varepsilon_{p}^{i}, d V\right)-\sigma_{t h}^{m}$ only applies when $\sigma_{I}^{i}\left(\varepsilon_{p}^{i}, d V\right)>\sigma_{t h}^{m}$ (where the principal stress is greater than the threshold stress), otherwise it is taken as 0 . The relation between current yield stress and reference yield stress enables the Bordet Model to account for the effects of strain rate and temperature. The addition of the threshold stress parameter allows for the removal from consideration of integration points where the stress is below this, and the addition of the $d \varepsilon_{p}^{i}$ means the Bordet Model only considers newly nucleated cracks, ignoring cracks from previous steps which would have blunted, and hence will not trigger brittle fracture.

The reference yield stress is calculated at the un-irradiated $T_{0}$ temperature for both the irradiated and un-irradiated states, and the integration point yield stress is calculated using the model temperature, assuming quasi-static loading and negligible strain rate effects.

\subsubsection{WST Model}

For the WST model the cumulative probability of failure for an elementary volume in a finite element analysis is given by Eq. (2). The terms $f(r)$ and $p_{c, i}$ are the probability density of microdefect sizes and the probability of particle rupture, and are defined, when following the WST distribution of carbides, as

$$
f(r)=\frac{(\gamma-2)^{\gamma-1}}{r_{0} \Gamma(\gamma-1)}\left(\frac{r}{r_{0}}\right)^{-\gamma} \exp \left(-(\gamma-2) \frac{r_{0}}{r}\right)
$$

where $r_{0}$ is the average particle size, $r$ is the particle size being considered and $\gamma$ is a shape parameter describing the particle size distribution,

$$
p_{c, i}=1-\exp \left[-\left(\frac{r}{r_{0}}\right)^{3}\left(\frac{\sigma_{\text {part }}}{\sigma_{0}}\right)^{m}\right]
$$

where $\sigma_{\text {part }}$ is an energy term calculated from the matrix principal stress and total principal strain, and the particle modulus of elasticity, $\sigma_{0}$ is a reference stress used for calibration, and $m$ is a calibration constant.

$$
\sigma_{\text {part }}=\sqrt{1.3 \sigma_{\text {matrix }} \varepsilon_{\text {matrix }} E_{\text {part }}}
$$


The critical crack size $r_{c}$ is calculated using the Griffith criterion. For a penny-shaped crack the criterion provides the following relationship between $r_{c}$ and the stress $\sigma_{\alpha}$ acting normal to the microcrack plane:

$$
r_{c}=\frac{\pi E\left(\gamma_{s}+\gamma_{p}\right)}{2\left(1-v^{2}\right) \sigma_{\alpha}^{2}}
$$

where $\gamma_{s}$ is the surface energy of the matrix, $\gamma_{p}$ is the plastic work associated with crack propagation, and $E$ is the matrix Young's modulus of elasticity.

\subsection{Developed Model}

The expression for the probability of particle failure is based on experimental observations that this probability depends not only on the mechanical fields but also on the particle size, similarly to the WST model [16]. The rationale is that larger particles have higher probability of failure than smaller particles under identical mechanical conditions, and the larger particles release more energy when they fail, so are more likely to rupture the surrounding matrix and cause cleavage fracture.

The particles are assumed to be elastic-brittle with elastic constants equal to those of the matrix as a first approximation. The deformation of a particle is assumed to follow the deformation of the matrix as far as the particle remains intact. Hence the principal strains in the intact particle, which are elastic, equal the total principal strains in the surrounding matrix. The principal stresses in the particle, denoted by $\Sigma_{\alpha}$, are governed by Hook's law

$$
\Sigma_{\alpha}=\frac{E}{1+v}\left(\varepsilon_{\alpha}^{e}+\varepsilon_{\alpha}^{p}\right)+\frac{E v}{(1+v)(1-2 v)} \varepsilon_{\alpha \alpha}^{e}, \quad \alpha=1,2,3
$$

where $E$ is Young's modulus and $v$ is Poisson's ratio for the particle and the matrix, and $\varepsilon_{\alpha \alpha}^{e}$ denotes the volumetric strain, $\varepsilon_{\alpha \alpha}^{e}=\varepsilon_{1}^{e}+\varepsilon_{2}^{e}+\varepsilon_{3}^{e}$, in the particle. Note that the plastic incompressibility condition, $\varepsilon_{\alpha \alpha}^{p}=\varepsilon_{1}^{p}+\varepsilon_{2}^{p}+\varepsilon_{3}^{p}=0$, is taken into account in (14). This can be written in terms of the principal stresses and principal plastic strains in the matrix, $\sigma_{\alpha}$ and $\varepsilon_{\alpha}^{p}$ respectively, with

$$
\Sigma_{\alpha}=\sigma_{\alpha}+\frac{E}{1+v} \varepsilon_{\alpha}^{p}, \quad \alpha=1,2,3
$$

which expresses the effect of the plastic overload on the particle. Further, the elastic strain energy density $\psi$ in the particles can be written in the form

$$
\psi=\frac{1+v}{2 E}\left(\Sigma_{1}^{2}+\Sigma_{2}^{2}+\Sigma_{3}^{2}\right)-\frac{v}{2 E} \Sigma_{\alpha \alpha}^{2}
$$

where $\Sigma_{\alpha \alpha}$ denotes the hydrostatic stress, $\Sigma_{\alpha \alpha}=\Sigma_{1}+\Sigma_{2}+\Sigma_{3}$, in the particle, which equals the hydrostatic stress in the matrix due to incompressibility. The energy density associated with particle failure normal to the maximum principal stress is derived from the expression $\Sigma_{1} \cdot \partial \psi / \partial \Sigma_{1}$ [21], and after minor algebraic manipulation is given by

$$
\psi_{1}=\frac{1+v}{E} \Sigma_{1}^{2}-\frac{v}{E} \Sigma_{1} \Sigma_{\alpha \alpha}
$$

If a particle of size $r$ ruptures normally to the maximum principal stress upon achieving some critical condition, the energy lost (or the work of rupture) will be proportional to $\Psi_{c}=r^{3} \psi_{c}$. The rupture of a 
particle is a stochastic event governed by the work of rupture and depending on the unknown particle constitution. The survival probability, $p_{s}$, of the particle must decrease with increasing work of rupture, which can be written in the form

$$
\frac{d p_{s}}{p_{s}}=-\frac{d \Psi_{c}}{\Psi_{0}}
$$

where $\Psi_{0}$ is a scaling energy. The probability of a particle rupturing the matrix, $p_{c}$, is one minus the survival probability

$$
p_{c}=1-\exp \left(-\frac{\Psi_{c}}{\Psi_{0}}\right)=1-\exp \left[-\left(\frac{r}{r_{0}}\right)^{3} \frac{\psi_{c}}{\psi_{0}}\right]
$$

where the rupture energy density scale $\psi_{0}=\Psi_{0} / r_{0}^{3}$ is introduced for convenience. Note that Eq. (19) incorporates the effects of plastic strain and stress triaxiality. Increased plastic strains in the matrix will result in increased $\psi_{c}$ and hence probability of micro-crack formation, with all other conditions equal. Inversely, increased hydrostatic stress, will result in a corresponding reduction in the probability of nucleation, lead to reduced $\psi_{c}$ and hence reduced probability of micro-crack formation under equal other conditions, see Eqs. (15) and (17).

Equations (17) and (19) are used in Eq. (2) to calculate individual probabilities of failure at the integration points by numerical integration. The lower limit of the integral, i.e. the critical micro-crack size at given point, is defined with a heuristic argument leading to a new modification of the Griffith criterion to account for plasticity. The behaviour of a micro-crack of radius $r$ formed in the plastic matrix is assumed to correspond to a fictitious micro-crack of radius $r_{f}>r$ in an elastic matrix. The crack opening displacement of a penny-shaped crack of radius $r_{f}$ in an elastic material subject to normal stress $\sigma_{I}$ is given by

$$
u(x)=\frac{4\left(1-v^{2}\right) \sigma_{I}}{\pi E} \sqrt{r_{f}^{2}-x^{2}}, \quad 0<x<r_{f}
$$

The blunting of the physical micro-crack formed after particle rupture can be approximated by $r \varepsilon_{I}^{p}$. The fictitious micro-crack size is then defined in such a way that the opening at $x=r$ equals the blunting of the physical micro-crack, i.e. $u(r)=r \varepsilon_{I}^{p}$. Solving Eq. (20) with this constraint leads to the following expression for the fictitious micro-crack size

$$
r_{f}=r \sqrt{1+\left[\frac{\pi E \varepsilon_{I}^{p}}{4\left(1-v^{2}\right) \sigma_{I}}\right]^{2}}
$$

Used in conjunction with the Griffith criterion an effective critical micro-crack size can be defined that accounts for the crack tip blunting as

$$
r_{c}=\frac{\pi E \gamma_{s}}{2\left(1-v^{2}\right) \sigma_{I}^{2}} \sqrt{1+\left[\frac{\pi E \varepsilon_{I}^{p}}{4\left(1-v^{2}\right) \sigma_{I}}\right]^{2}}
$$

The square-root factor in Eq. (22) replaces the exponent of plastic strain in the modified Griffith criterion used previously $[2,14]$. As before, it is intended to account for the reduction in crack driving force due to plastic dissipation in the matrix (i.e. crack tip blunting). The difference is that in the case of Eq. (22) the effect is not only dependent on the plastic strain but also on stress triaxiality via the ratio between the maximum plastic strain and the maximum stress. 


\section{Experimental Data}

One material has been considered here under both irradiated and un-irradiated states. The material considered is an RPV 22NiMoCr37 ferritic steel, known as Euro Material A, for which the mechanical and fracture toughness properties at a number of temperatures and irradiation states within the lower shelf and in DBT are available from the Euratom FP6 project PERFECT and FP7 project PERFORM60. The data available (from PERFORM 60 [22]) for this material includes the mechanical and low and high constraint fracture toughness properties (with initial $a_{0} / W=0.1$ and 0.5 respectively) over a range of temperatures and irradiation states within the lower shelf and in the DBT.

The fracture toughness properties were determined according to the ASTM standard [23] using $10 \mathrm{~mm}$ wide, $10 \mathrm{~mm}$ thick and $55 \mathrm{~mm}$ long pre cracked charpy V notch (PCCV) specimens in three-point bending. This standard is based on the Master Curve formalism, which defines the temperature dependence of a reference toughness, $K_{0}$, relative to a reference temperature, $T_{0}$, at which $K_{0}=108$ $\mathrm{MPa} \sqrt{\mathrm{m}}$, for high-constraint cracked geometries with reference crack front length $B_{0}=25.4 \mathrm{~mm}$

$$
K_{0}=31+77 \exp \left[0.019\left(T-T_{0}\right)\right]
$$

The scatter in measured cleavage fracture toughness values is described as a function of the probability of failure, $p$, and the actual crack front length, $B$, with

$$
K_{p}=K_{\min }+\left(K_{0}-K_{\min }\right)\left(\frac{B_{0}}{B}\right)^{1 / 4}\left(\ln \frac{1}{1-p}\right)^{1 / 4}
$$

where $K_{\min }$ is a temperature independent threshold toughness, $K_{\min }=20 \mathrm{MPa} \sqrt{\mathrm{m}}$ in [23]. Equations (23) and (24) define the cleavage fracture toughness behaviour of a material with known $T_{0}$. The Master Curve fit for the experimental data for each case is shown in Figure 1, where the experimental data is given by the circular data points, the 50 percent prediction of toughness by the solid curve and the $5^{\text {th }}$ and $95^{\text {th }}$ percentile predictions by the two dashed curves.

From these data the reference temperature for the un-irradiated high constraint $\left(a_{0} / W=0.5\right)$ specimens has been calculated as $T_{0}=-108{ }^{\circ} \mathrm{C}$. For the irradiated high constraint specimens $T_{0}=-79$ ${ }^{\circ} \mathrm{C}$, providing a fracture toughness shift of $\Delta T=30{ }^{\circ} \mathrm{C}$, for the un-irradiated low constraint specimens $\left(a_{0} / W=0.1\right) T_{0}=-153{ }^{\circ} \mathrm{C}$, providing a fracture toughness shift of $\Delta T=-44{ }^{\circ} \mathrm{C}$, and for the irradiated low constraint specimens $T_{0}=-126^{\circ} \mathrm{C}$, providing a fracture toughness shift of $\Delta T=-18$ ${ }^{\circ} \mathrm{C}$ [22]. These are tabulated in Table 1 along with the predicted shifts.

The temperature dependence of Young's Modulus, $E$, yield stress $\sigma_{y}$, and ultimate tensile strength $\sigma_{U T S}$ under un-irradiated conditions for Material A can be approximated as:

$$
\begin{aligned}
& E=-90 T+206000 \\
& \sigma_{y}=421.2+63.9 \exp (-T / 91) \\
& \sigma_{U T S}=564.1+70.2 \exp (-T / 108)
\end{aligned}
$$

where $T$ is in ${ }^{\circ} \mathrm{C}$ and $E, \sigma_{y}$, and $\sigma_{U T S}$ are in MPa. Poisson's ratio is $v=0.3$, and is independent of temperature. For the irradiated state considered here (with a total fluence of $4.3 \times 10^{19} \mathrm{n} \mathrm{cm}^{-2}$, En $>1$ $\mathrm{MeV}$ at $285^{\circ} \mathrm{C}$ ) $E$ is given by Eq. (25) while $\sigma_{y}$ and $\sigma_{U T S}$ are given by

$$
\begin{aligned}
& \sigma_{y}=490.4+62.1 \exp (-T / 95.2) \\
& \sigma_{U T S}=524.2+155.1 \exp (-T / 188.2)
\end{aligned}
$$


(A)

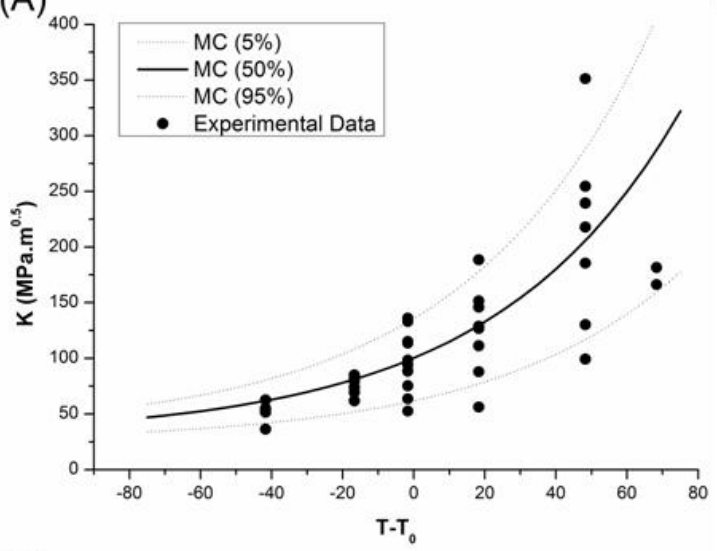

(C)

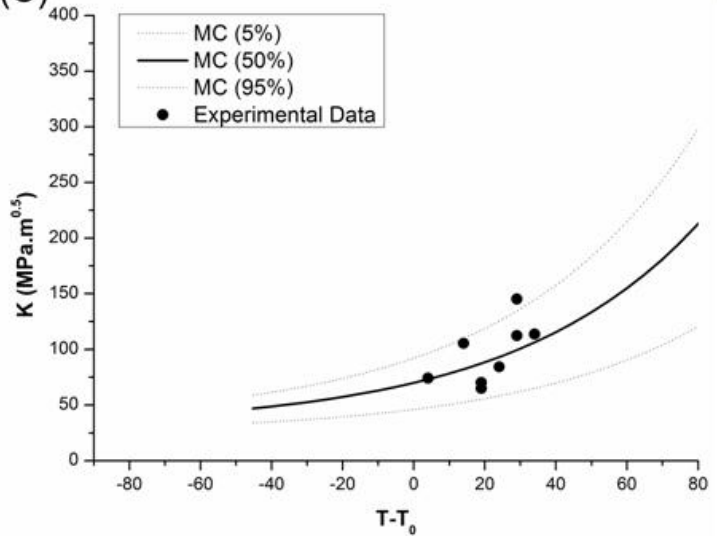

(B)

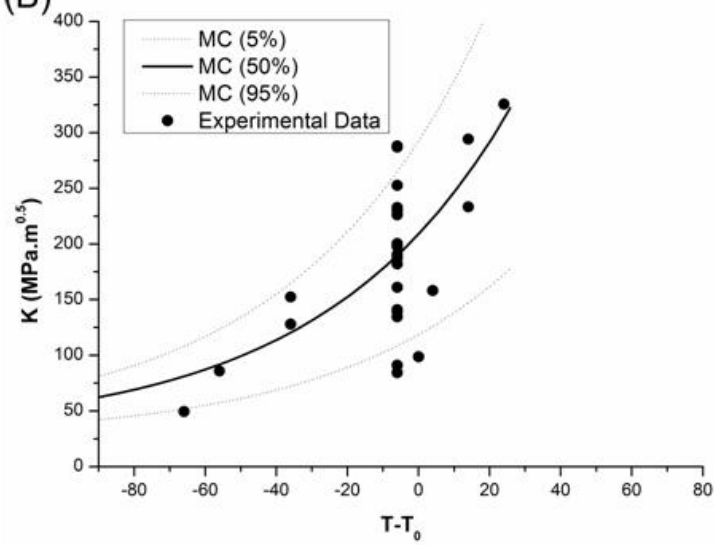

(D)

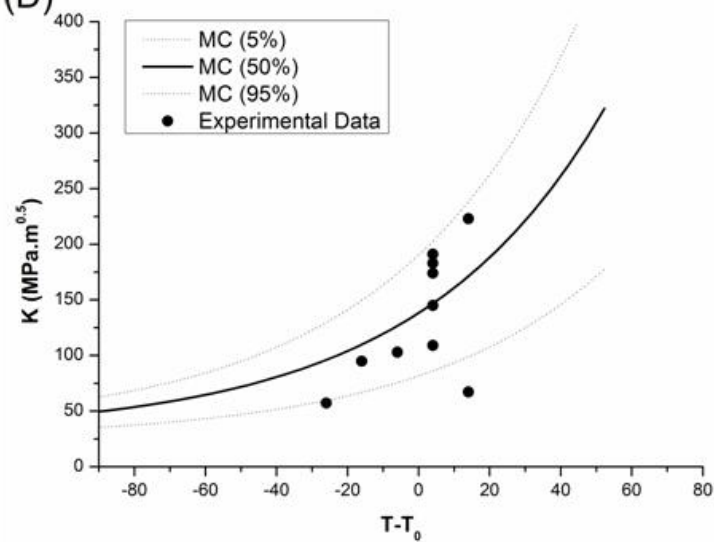

Figure 1. Master Curve (MC) representation and experimental data for: (A) Un-irradiated high constraint conditions, (B) Un-irradiated low constraint conditions, (C) Irradiated high constraint conditions and (D) Irradiated low constraint conditions

The density and size distribution of cleavage initiating particles for Material A have been reported in [24] . For Material A the nature of the initiators has been determined by fractography revealing that cleavage in this material initiated predominantly at metal carbides (specifically M3C and M23C6). Comprehensive metallographic examination of carbides provided number density $\rho=7.6 \times 10^{17} \mathrm{~m}^{-3}$ and a probability density of particle sizes which was fit by

$$
f(r)=\frac{\beta}{r_{0}}\left(\frac{r}{r_{0}}\right)^{-\beta-1} \exp \left[-\left(\frac{r}{r_{0}}\right)^{-\beta}\right]
$$

with the shape parameter, $\beta$, equal to 2.7 , and the scale parameter representing the mean particle size, $r_{0}$, equal to $0.036 \mu \mathrm{m}$.

\section{Finite Element Model}

A three-point bend specimen model was used in the finite element analyses to which the developed LA model has here been applied, as this specimen type most closely resembles the Pre-Cracked Charpy-V (PCCV) specimens used in the tests. Three crack depths have been considered, one to represent high and two to represent low constraint conditions; with crack depths of $a / t=0.5,0.2$ and 0.1 respectively. Two low constraint crack depths were considered to encompass the possible range of crack lengths at which cleavage failure occurred when allowing for small levels of observed ductile crack extension prior to failure. For brevity the results of the analyses using $a / t=0.2$ are detailed fully herein and are referred to as the low constraint condition, apart from a brief discussion of the results of the $a / t=0.1$, as the results were seen to follow similar trends.

The three point bend specimens used were $10 \mathrm{~mm}$ through thickness by $55 \mathrm{~mm}$ long and $10 \mathrm{~mm}$ 
deep to match the fracture tests within a three-dimensional finite element analyses. Non-deforming, rigid, loading rollers were positioned with a total span of $40 \mathrm{~mm}$ (i.e. $7.5 \mathrm{~mm}$ from the edges of the specimen) and at the centre crack back-face. The finite element model to describe this geometry was created and run within ABAQUS 6.11 [25] and was composed of up to 37,000 C3D8 three-dimensional elements. The crack tip region was modelled as a fine blunt notch with a crack-tip radius of $25 \mu \mathrm{m}$. The blunt notch was used to allow the use of large strain analysis, which is necessary to provide the correct stress field ahead of the crack tip. The level of blunting was held constant at this value, chosen to be representative of the blunting present at fracture in the middle of the transition region, to avoid introducing an additional variable into the analysis which would require a different mesh for each temperature modelled. The mesh thickness increased from $0.054 \mathrm{~mm}$ at the surface of the specimen to $1.6 \mathrm{~mm}$ at the centre to capture the transition from plane stress to plane strain dominated regions. An illustration of the finite element model can be seen in Figure 2 as the global model which also incorporates the crack tip region.

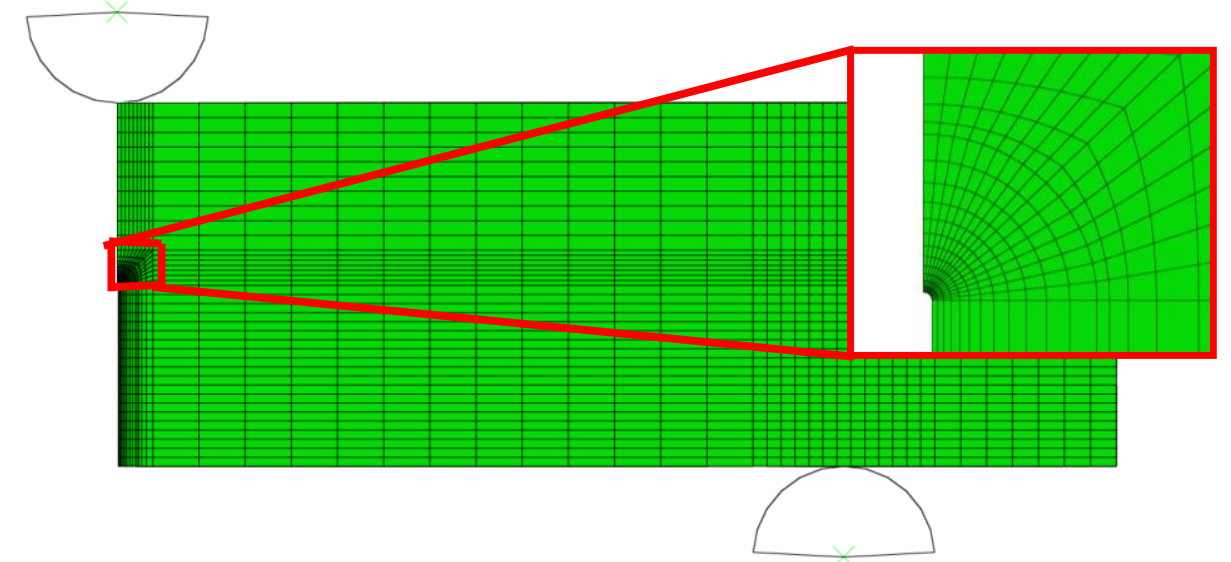

Figure 2. FE Model of 3 point bend specimen (side view of three-dimensional model)

The primary loading was applied under displacement control conditions by displacing the bottom loading pin into the specimen whilst restraining the top pin. The symmetry of the geometry and loading allowed the un-cracked ligament to act as the symmetry plane. The symmetry of the through thickness allowed the centre of the specimen perpendicular to the crack front to also act as a symmetry plane. Boundary conditions were applied to the loading pins to prevent free rotation and unwanted displacement. Standard contact was used with a small adjustment of $0.015 \mathrm{~mm}$ to the mesh around the rigid surface of the loading pins to ensure correct transference of the load. The contact was also modelled as having no separation, ensuring contact throughout the analyses.

In each analysis the estimate of the $J$-contour integral was used to provide the value of crack tip stress intensity factor $K$, which was extracted as part of the post-processing routine used in the local approach prediction of failure. However, it is noted that the blunt notch was used to accommodate large strain analyses with large deformations at the crack tip and that the plastic zone can become very large. To encompass as much of the plastic zone as possible $J$ was calculated up to 80 contours away from the crack tip node set (i.e. those on the blunt tip), which allowed the estimate of $J$ to cover as much of the plastic zone as possible whilst remaining contained in the model. Validation of the model was performed that provided confidence in the model, loading and extracted values of $J$. Values of the $J$-contour integral were taken at each loading increment in the analyses and converted to a value of $K$. All estimates of $J$ were taken at the deepest point of the crack and at the centre of the specimen to maintain plane-strain conditions.

The materials used in the finite element analyses were based upon Material A as presented in Section 3 over the range of temperatures in the irradiated and un-irradiated states. To interpret the yield stress and ultimate tensile strength within the analyses at a given temperature a power-law relation was used for the uniaxial stress, $\sigma$, and strain, $\varepsilon$, which remained elastic below yield and was dependent on $\sigma^{n}$ after yielding. The determination of the power law exponent, $n$, has been made using the Considère's rule describing the stress-strain condition at the point of loss of stability, represented by $\sigma_{U T S}$ in engineering stress terms. For both irradiation conditions a range of temperatures were assessed to cover the transition curve. These were generally spaced between $\pm 70{ }^{\circ} \mathrm{C}$ of the high constraint un- 
irradiated $T_{0}$ temperature in a maximum of $10{ }^{\circ} \mathrm{C}$ increments, giving a range in $n$ of 21.35 to 10.58 for the un-irradiated condition from $T_{0}-70^{\circ} \mathrm{C}$ to $T_{0}+70^{\circ} \mathrm{C}$, and 27.96 to 11.86 for the irradiated condition over the same temperature range.

\section{Results and discussion}

\subsection{Application of Microstructurally Informed Model to Material A}

For known carbide distribution parameters, $\beta$ and $r_{0}$, in principle the model requires a single calibration of the rupture energy density scale, $\psi_{0}$, in Eq. (Error! Reference source not found.). This can be performed by comparing experimentally measured positions of cleavage initiation sites with the profile of the individual failure probability ahead of the crack, calculated using Eq. (2) with Eqs. (Error! Reference source not found.) and (Error! Reference source not found.). By initially keeping the surface energy value, $\gamma_{s}$, set to the approximate value of $2.4 \mathrm{Jm}^{-2}$ for typical ferritic steels in the literature [26] and adopting the measured values for $\beta$ and $r_{0}$ the value of $\psi_{0}$ to best fit initiation sites was $\psi_{0}=10^{4}$. This was then used for all cases considering Material A. To scale the fracture data to match the experimental results $\gamma_{s}$ was calibrated (keeping $\psi_{0}=10^{4}$ ) at a single temperature, $T_{0}$ for the un-irradiated material, so that at applied $K=100.3 \mathrm{MPa} \sqrt{\mathrm{m}}$ the model predicted 50 percent probability of cleavage. The calibrated value for $\gamma_{s}$ was $2 \mathrm{Jm}^{-2}$. Note that the calibrated $\gamma_{s}$ is therefore within 20 percent of the literature value. It is also emphasised that the parameters were not modified when changing the temperature, constraint or irradiation; the only change was the $\sigma, \varepsilon$ relationship.

Figure 3 shows the experimental and predicted failure probability profiles as functions of normalised distance ahead of the crack obtained with the calibrated values of $\psi_{0}$ and $\gamma_{s}$. The green stars show experimentally measured locations of cleavage initiation sites reported in [14] for SEN(B) specimens with deep cracks tested to fracture at several temperatures. The results are ranked and normalised by the crack tip opening displacement, CTOD, so that the maximum individual failure probability is at unity and can be represented by a smooth curve. The CTOD was obtained from the FE model at the $T_{0}$ temperature at a load at which the applied $K=100.3 \mathrm{MPa} \sqrt{\mathrm{m}}$ by measuring the displacement of a node located at a 45 degree angle behind the crack tip. Clearly, the model captures the peak location of initiating particles accurately and approximates the overall shape sufficiently well.

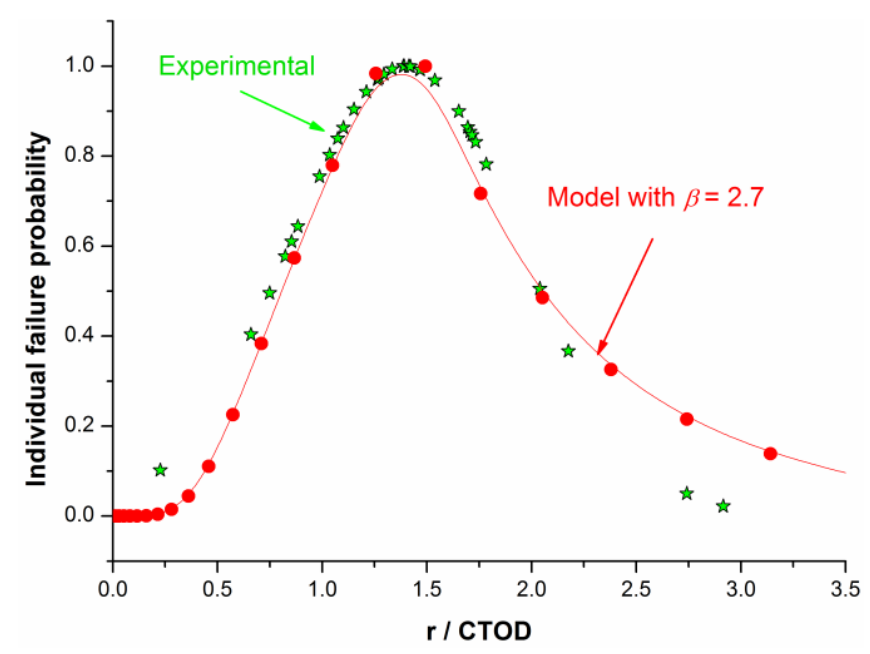

Figure 3. Individual failure probability predicted by Developed Model

The predictive bounds and shifts in $T_{0}$ from the model compared to experimental data are given in Figure 4 and Table 1 respectively for both the un-irradiated and irradiated materials for high and low constraint conditions using the same model parameters, varying only due to differences in $\sigma$ and $\varepsilon$.

The model slightly over predicts the fracture toughness values compared to data from tests from the lowest temperatures for the un-irradiated high constraint case. The probability bounds widen at higher temperatures, which is likely to be an effect of plasticity, which could manifest in both the accuracy of the finite element analyses and of the assumptions in the local approach model. 
The un-irradiated low constraint predicted values follow the test data for the lowest temperatures up to $40{ }^{\circ} \mathrm{C}$ below the $T_{0}$ temperature; however it predicts that cleavage failure will not occur at temperatures greater than this. If assessed in terms of a shift in the Master Curve determined $T_{0}$ value, the predicted shift in $T_{0}$ is $-37{ }^{\circ} \mathrm{C}$, which is a small under prediction of the magnitude of the shift compared to the observed shift of $-44^{\circ} \mathrm{C}$.

The predicted toughness curve is flatter than the experimental data for the irradiated high constraint case, although the data points are all within the probability bounds and the 50 percent probability passing through the centre of these points. The probability bounds are narrower here than for the un-irradiated high constraint case, most likely due to the irradiated material undergoing less plastic deformation. The predicted shift in $T_{0}$ is $25^{\circ} \mathrm{C}$, which is close to the observed shift of $30{ }^{\circ} \mathrm{C}$.

The irradiated low constraint predicted values over predict the toughness and have very wide probability bounds. However, if the predictions were shifted across by $30^{\circ} \mathrm{C}$ they would pass through the centre of the test data points, and match the test data gradient well. The predicted shift in $T_{0}$ is $-22{ }^{\circ} \mathrm{C}$, which is almost the same as the observed shift of $-18^{\circ} \mathrm{C}$.

(A)

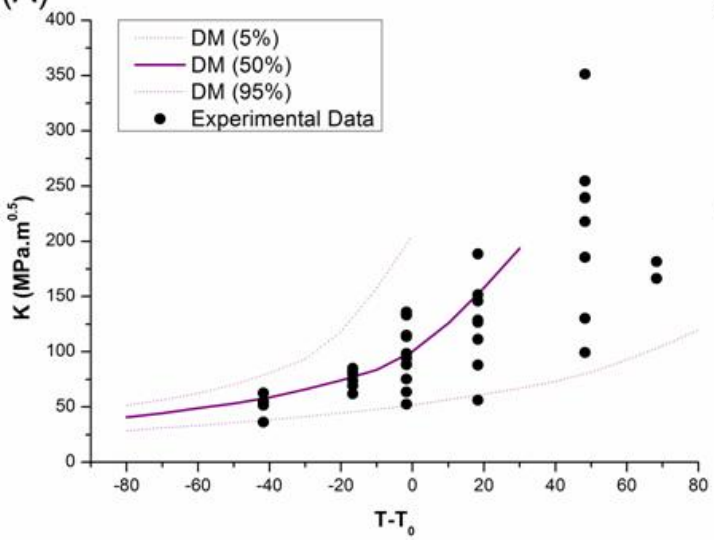

(C)

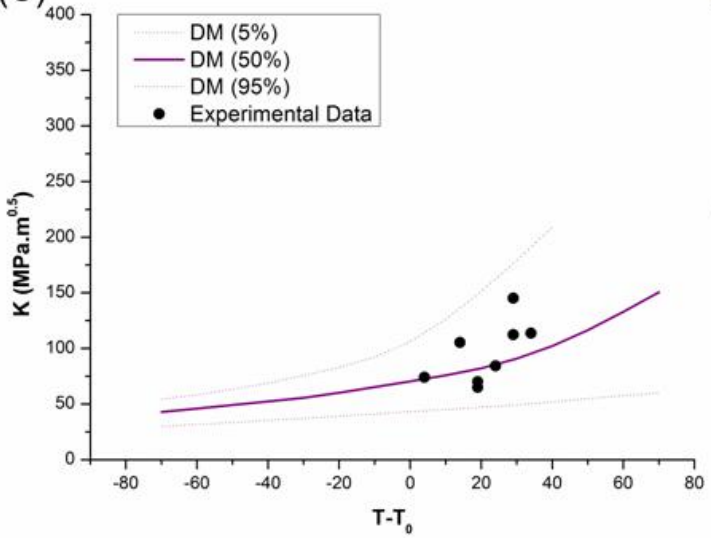

(B)

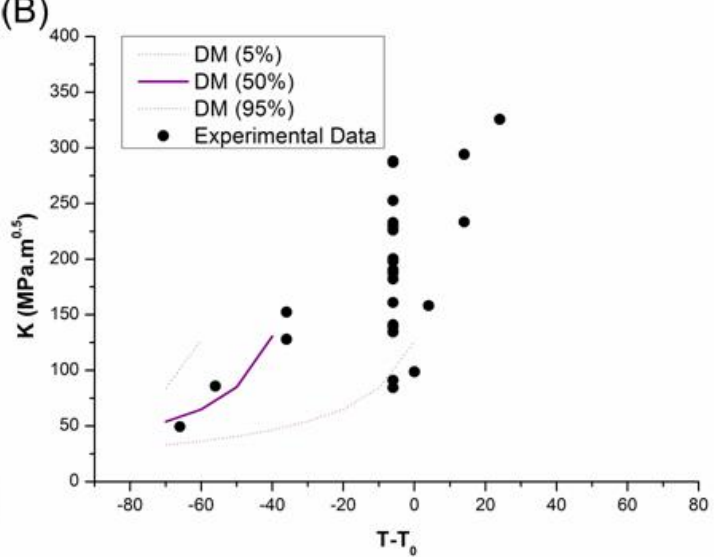

(D)

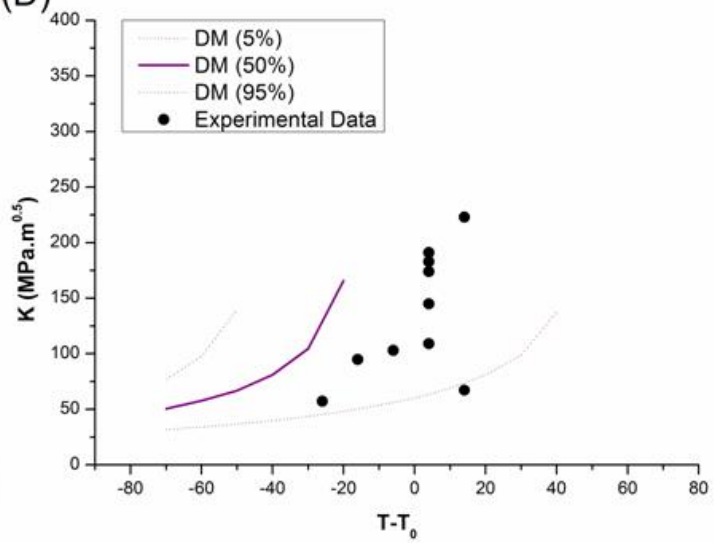

Figure 4. Temperature dependence predicted by Developed Model (DM), given by equations Error! Reference source not found. to Error! Reference source not found. when compared to experimental data for: (A) Un-irradiated high constraint conditions, (B) Un-irradiated low constraint conditions, (C) Irradiated high constraint conditions and (D) Irradiated low constraint conditions.

As the un-irradiated prediction of toughness was a little higher than the test data at the same temperature the Developed Model was calibrated a second time, altering $\gamma_{s}$ iteratively so that at applied $K=100.3 \mathrm{MPa} \sqrt{\mathrm{m}}$ the model predicted 50 percent probability of cleavage at a temperature of $T_{0}+10{ }^{\circ} \mathrm{C}$. This was done to investigate the possible effects of a misleading calibration from that when assuming an incorrect Master Curve $T_{0}$. The results of this are shown in Figure 5, with the predicted toughness values fit the data better for both of the un-irradiated cases and for the irradiated 
low constraint case. The model slightly under predicts the toughness for the irradiated high constraint case, but all but one of the experimental data points are still within the probability bounds. 
Table 1. Summary of the predicted and observed shifts in $T_{0}$ using the Developed Model

\begin{tabular}{|l|c|c|c|}
\hline & Observed $\mathrm{T}_{0}\left({ }^{\circ} \mathrm{C}\right)$ & Observed shift $\left({ }^{\circ} \mathrm{C}\right)$ & Developed Model shift $\left({ }^{\circ} \mathrm{C}\right)$ \\
\hline $\begin{array}{l}\text { Un-irradiated High } \\
\text { Constraint }\end{array}$ & -108 & 0 & 0 \\
\hline $\begin{array}{l}\text { Un-irradiated Low } \\
\text { Constraint }\end{array}$ & -153 & -44 & -37 \\
\hline $\begin{array}{l}\text { Irradiated High } \\
\text { Constraint }\end{array}$ & -79 & +30 & +25 \\
\hline $\begin{array}{l}\text { Irradiated Low } \\
\text { Constraint }\end{array}$ & -126 & -18 & -22 \\
\hline
\end{tabular}

(A)

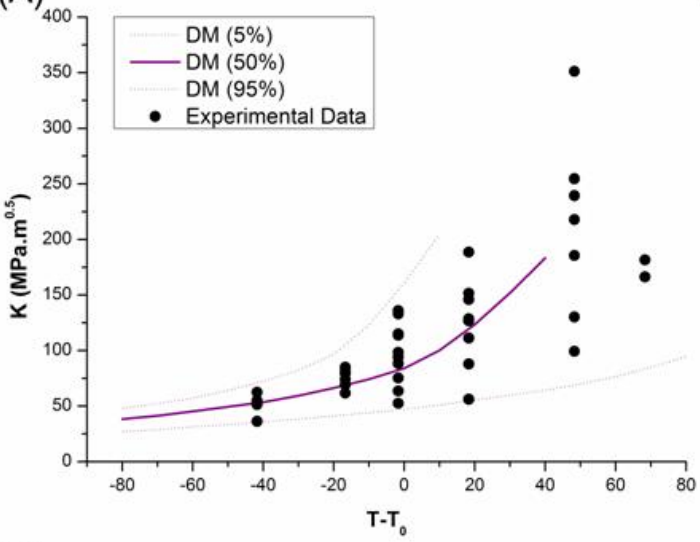

(C)

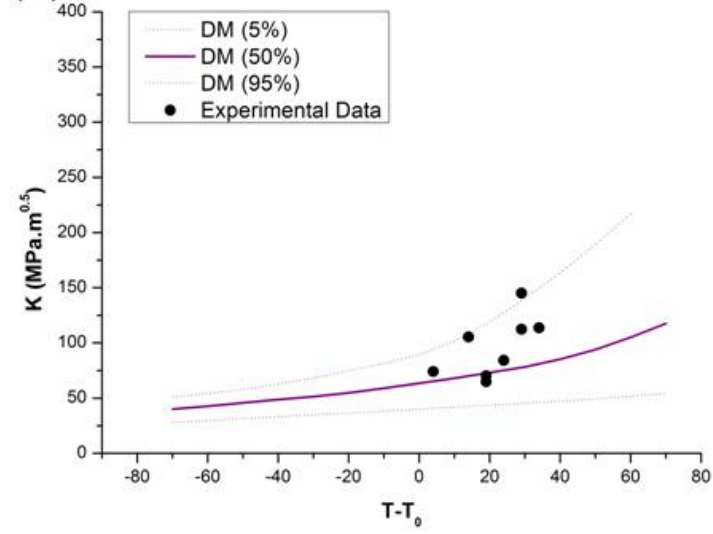

(B)

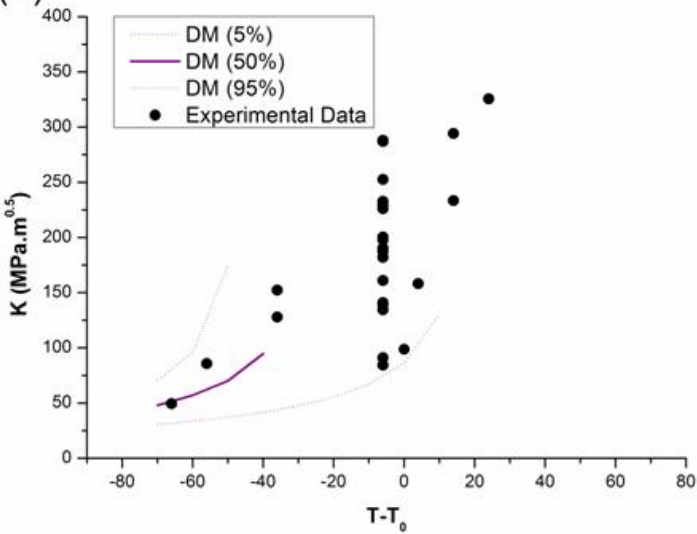

(D)

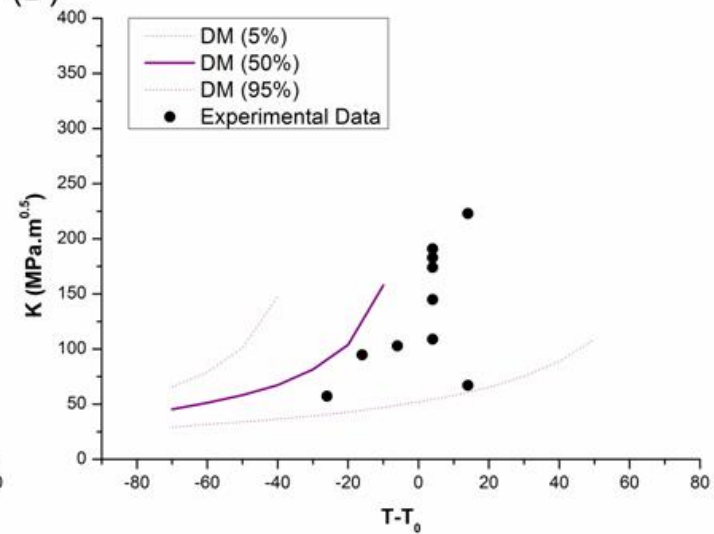

Figure 5. Temperature dependence predicted by Developed Model (DM), given by equations Error! Reference source not found. to Error! Reference source not found., calibrated with $\boldsymbol{T}_{\boldsymbol{0}}$ shifted

by $+10{ }^{\circ} \mathrm{C}$ when compared to experimental data for: (A) Un-irradiated high constraint conditions, (B) Un-irradiated low constraint conditions, (C) Irradiated high constraint conditions and (D) Irradiated low constraint conditions

\subsection{Application of Existing Local Approach Models to Material A}

Predictions for the modified Beremin model, Bordet model and the WST model for both unirradiated and irradiated materials in high and low constraint conditions are shown in Figures 6-8 respectively. The predicted shifts in $T_{0}$ are shown in Table. The calibration of each model is discussed in Appendix A.

It is noted that each of these models can be implemented with temperature dependent variables, where the Modified Beremin model can vary $\sigma_{u}$ with temperature, the WST model can vary $\gamma_{p}$ with 
temperature and the Bordet model calculates a temperature and material dependent $\sigma_{u}$ using empirically obtained constants to improve their predicted fracture toughness curves. However, these were not varied as this investigation calibrates the different approaches at a single temperature in the un-irradiated condition with high constraint, so the predictions of change in fracture toughness due to irradiation and changes in constraint use only the changes in tensile properties and FEA model geometry.

(A)
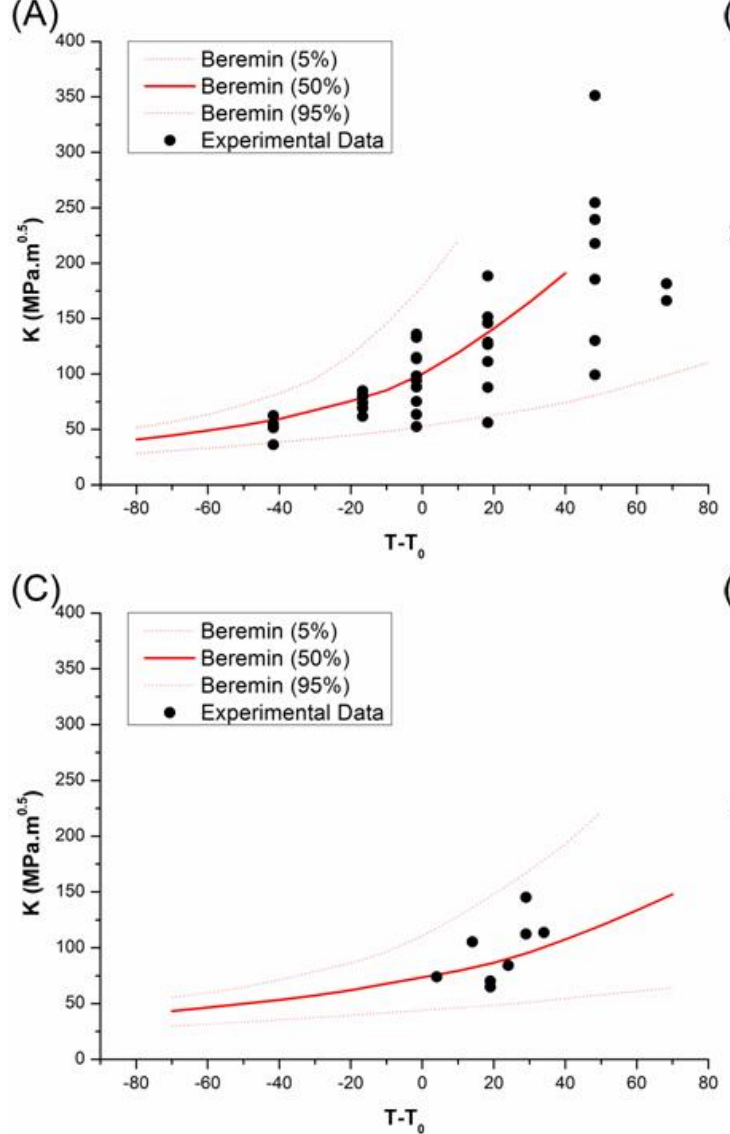

(B)

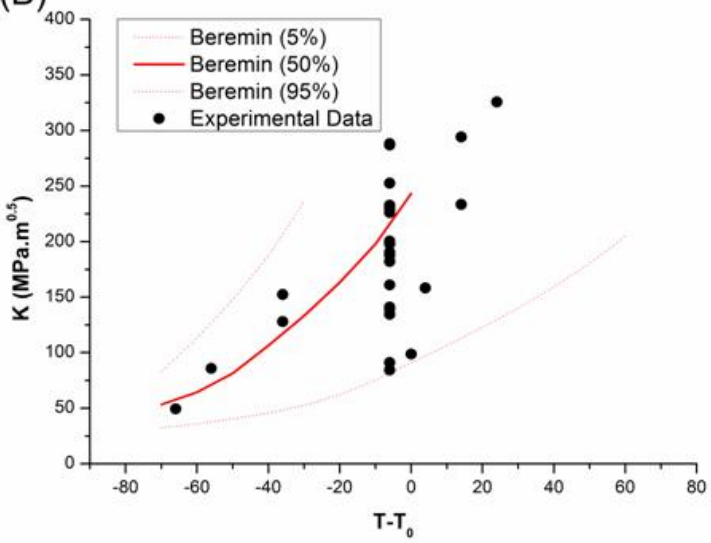

(D)

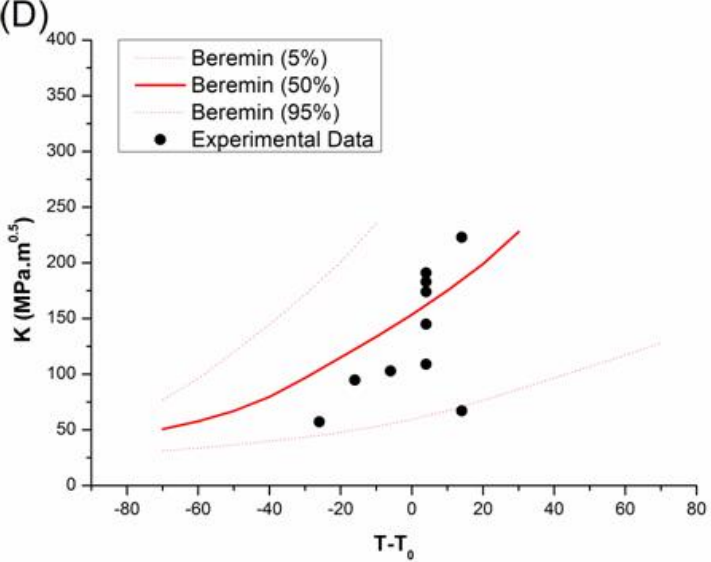

Figure 6. Temperature dependence predicted by Beremin model calibrated at $T_{0}$ when compared to experimental data for: (A) Un-irradiated high constraint conditions, (B) Un-irradiated low constraint conditions, (C) Irradiated high constraint conditions and (D) Irradiated low constraint conditions.

\subsubsection{Modified Beremin model}

The Modified Beremin model shows broad agreement with the experimental data for the unirradiated high constraint case, slightly over predicting the toughness, but capturing the change in toughness values with temperature and containing almost all of the experimental data points within the probability bounds. The experimental data are also within the bounds and the curve accurately describes the change in toughness with temperature.

When applied to the un-irradiated low constraint case the model partially captures the change in constraint, with a definite upswing as the temperature increases. This change in upswing is steeper than that of the Master Curve and would appear to over predict the toughness at higher temperatures when compared to the experimental data. The predicted shift in $T_{0}$ for this low constraint case is under predicted at $-35^{\circ} \mathrm{C}$, compared to the observed shift of $-44{ }^{\circ} \mathrm{C}$.

The predicted fracture toughness curve for the irradiated high constraint case appears to fit the data well, which is just contained within the probability bounds. The predicted shift in $T_{0}$ is $22^{\circ} \mathrm{C}$, which is a small under prediction compared to the observed shift of $30^{\circ} \mathrm{C}$.

The model appears to account for the combined effects of low constraint and irradiation well, although it has very wide probability bounds. The predicted shift in $T_{0}$ of $-21{ }^{\circ} \mathrm{C}$ is close to the observed shift of $-18^{\circ} \mathrm{C}$. The data are all within the probability bounds of the model. 
(A)

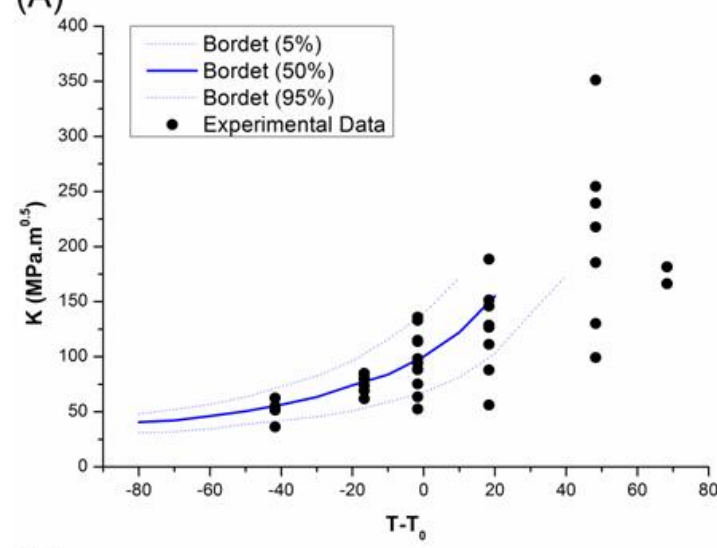

(C)

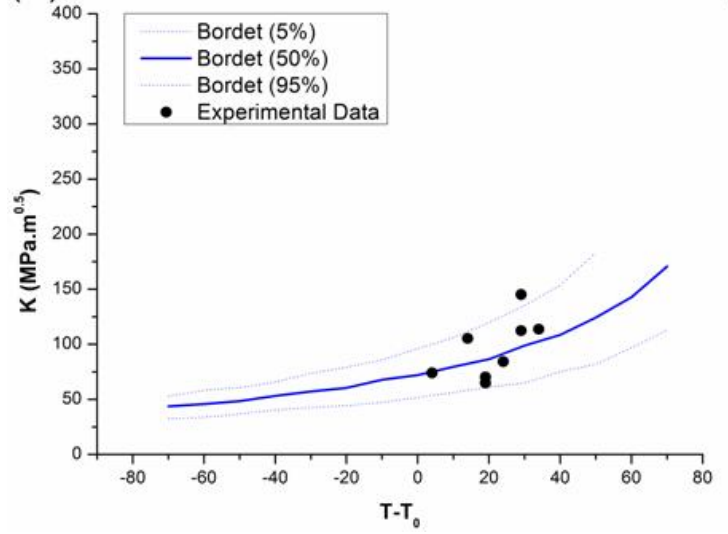

(B)

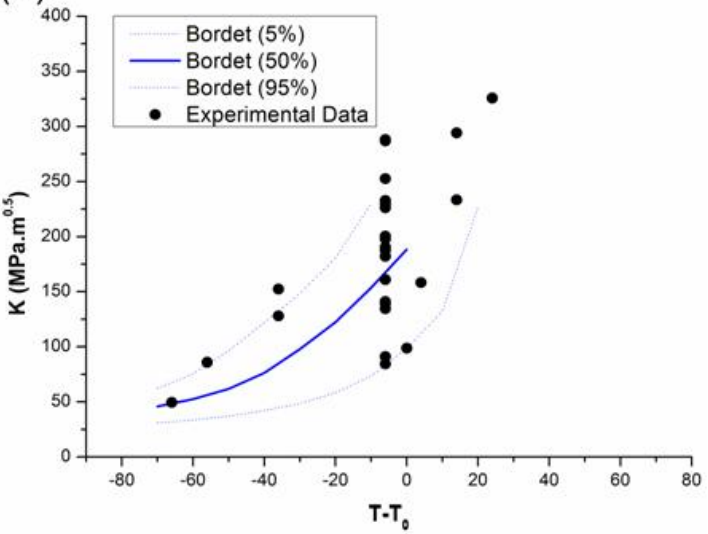

(D)

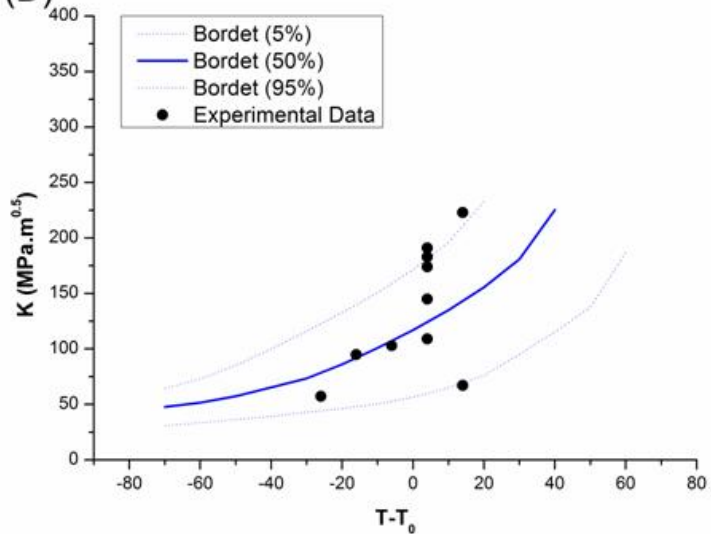

Figure 7. Temperature dependence predicted by Bordet model calibrated at $T_{0}$ when compared to experimental data for: (A) Un-irradiated high constraint conditions, (B) Un-irradiated low constraint conditions, (C) Irradiated high constraint conditions and (D) Irradiated low constraint conditions.

\subsubsection{Bordet model}

The Bordet model also over predicts the toughness values compared to the experimental data, and predicts that cleavage fracture will not occur at temperatures greater than the $T_{0}$ temperature. Although it does capture the change in toughness values with temperature, many of the experimental data points fall below the 5 percent probability bound, as the bounds are much narrower than the data would suggest.

For the un-irradiated low constraint case, the model prediction is steeper and lower than the experimental data, and appears to under predict the toughness more for lower temperatures, with several of the lowest temperature data points above the upper probability bound. The predicted shift in $T_{0}$ is $-22{ }^{\circ} \mathrm{C}$, which is a large under prediction of the magnitude of the shift compared to the observed shift of $-44^{\circ} \mathrm{C}$.

With the irradiated high constraint cases the model captures the experimental data well, although is perhaps slightly flatter, with a small over-prediction of toughness at low temperatures and an under prediction of toughness for high temperatures. This is reflected in the small over prediction of the shift in $T_{0}$ of $35^{\circ} \mathrm{C}$, compared with the observed shift of $30^{\circ} \mathrm{C}$.

The model appears to partially account for the change in constraint effects for the low constraint irradiated condition. The prediction fails to completely capture the upswing in the data, and several data points exceed the 95 percent probability bounds. It also under predicts the shift in $T_{0}$, with a predicted shift of $-6{ }^{\circ} \mathrm{C}$ compared to the observed shift of $-18{ }^{\circ} \mathrm{C}$. 

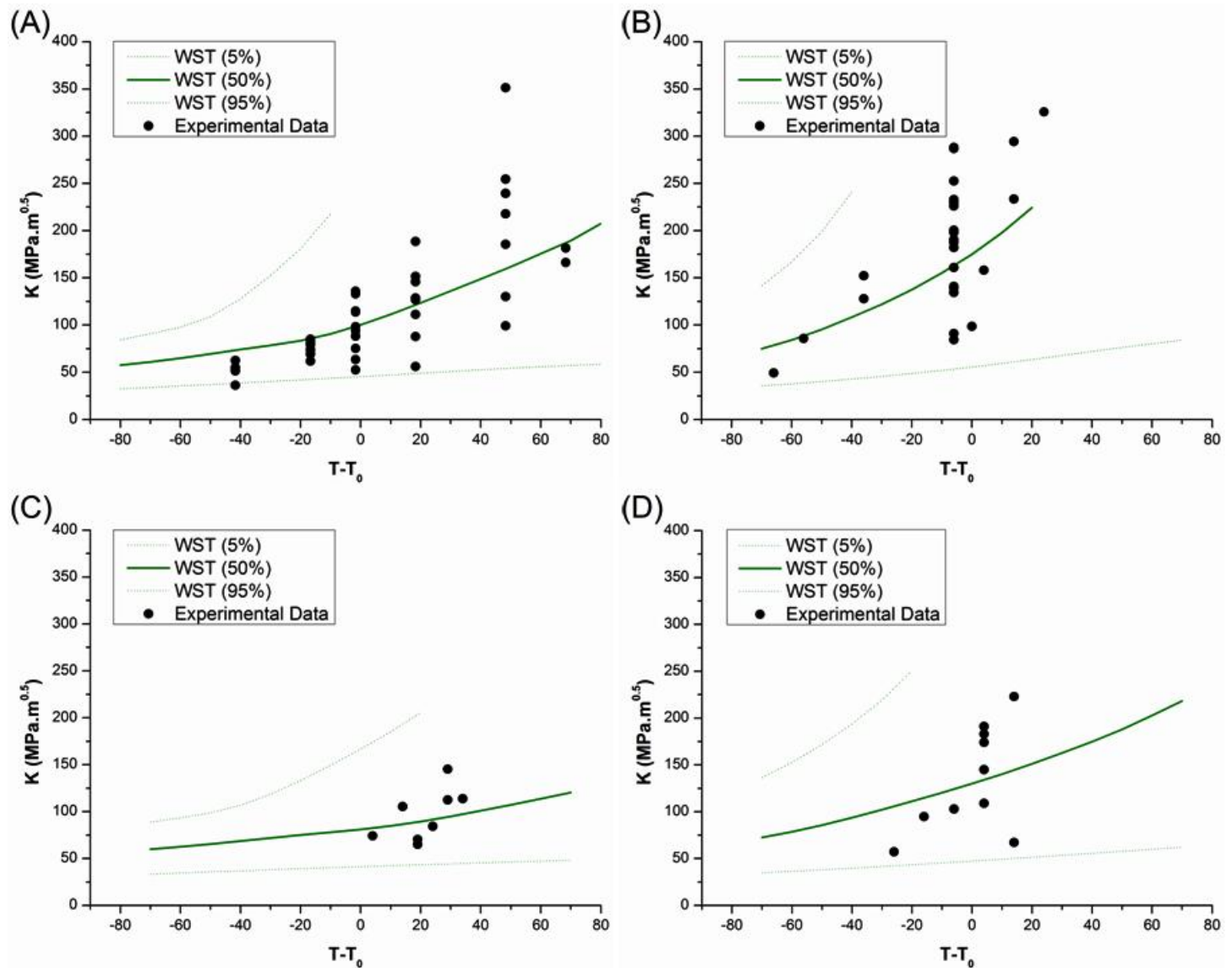

Figure 8. Temperature dependence predicted by WST model calibrated at $T_{0}$ when compared to experimental data for: (A) Un-irradiated high constraint conditions, (B) Un-irradiated low constraint conditions, (C) Irradiated high constraint conditions and (D) Irradiated low constraint conditions.

\subsubsection{WST model}

The WST prediction curve captures the trend of the change in toughness values with temperature, although it is somewhat flatter than the experimental data points for the un-irradiated high constraint case, slightly over predicting the low temperature toughness values and under predicting the high temperature values, with wide probability bounds.

The model does capture some of the upswing for the un-irradiated low constraint case, although it is still less steep than the data. It predicts the shift in $T_{0}$ of $-44{ }^{\circ} \mathrm{C}$, which is the same as the observed shift.

The predicted toughness is flatter than the experimental data for the irradiated high constraint case. The data falls within the predicted probability bounds, although these are wider than the experimental data would indicate. This under prediction of the toughness at higher temperatures is reflected in the under prediction of the shift in $T_{0}$ of $14{ }^{\circ} \mathrm{C}$, compared to the observed shift of $30^{\circ} \mathrm{C}$.

This pattern is repeated for the low constraint irradiated case, where the predicted toughness not as steep as the data indicates, with the model over predicting the shift in $T_{0}$ as being $-38{ }^{\circ} \mathrm{C}$, compared to the experimentally determined $-18^{\circ} \mathrm{C}$.

Table 2. Summary of the predicted and observed shifts in $T_{0}$ using the other Local Approaches

\begin{tabular}{|l|c|c|c|c|}
\hline & $\begin{array}{c}\text { Observed shift } \\
\left({ }^{\circ} \mathrm{C}\right)\end{array}$ & $\begin{array}{c}\text { Modified } \\
\text { Beremin shift } \\
\left({ }^{\circ} \mathrm{C}\right)\end{array}$ & $\begin{array}{c}\text { Bordet } \\
\text { shift }\left({ }^{\circ} \mathrm{C}\right)\end{array}$ & $\begin{array}{c}\text { WST } \\
\text { shift }\left({ }^{\circ} \mathrm{C}\right)\end{array}$ \\
\hline $\begin{array}{l}\text { Un-irradiated } \\
\text { Low }\end{array}$ & -44 & -35 & -22 & -44 \\
\hline
\end{tabular}




\begin{tabular}{|l|c|c|c|c|}
\hline $\begin{array}{l}\text { Irradiated } \\
\text { High } \\
\text { Constraint }\end{array}$ & +30 & +22 & +35 & +14 \\
\hline $\begin{array}{l}\text { Irradiated Low } \\
\text { Constraint }\end{array}$ & -18 & -21 & -6 & -38 \\
\hline
\end{tabular}

\subsubsection{Low constraint conditions}

Two low constraint conditions were modelled, the first condition, which is reported here fully, using an $a / t=0.2$, the second with $a / t=0.1$. The results from the $a / t=0.1$ condition were seen to follow the same trends as the $a / t=0.2$ case but with the transition temperature increased further. This was because a higher load was required to provide the same stress at the crack tip, slightly reducing the probability of failure.

\subsubsection{Comparison of Approaches}

The results presented demonstrate that there is, on the whole, little difference between the predictions of the models tested. The most simple to implement is the Modified Beremin Model as this is simply based on a stress and is calibrated through three individual terms. The Bordet model is a more complex version of the Beremin model but requires six terms to be calibrated. The WST model and the Developed Model attempt to provide micro-structurally informed models; where the Developed Model only requires two terms $\left(\psi_{0}\right.$ and $\left.\gamma_{s}\right)$ to be calibrated when the distribution of initiating particles is known. This means that the Developed Model provides an approach where a minimal number of terms require calibration and in practice the surface energy of the matrix material can be taken from the literature.

For the Modified Beremin and Bordet models the $m$ term is nominally related to the distribution of brittle initiating features and links to $\beta$ roughly as $m=2 \beta-2$. Here the values of $m$ which gave the best fitting curves compared to experimental data and their $\beta$ equivalent (in brackets) in the Modified Beremin and Bordet model were both 19 (10.5). Clearly these results are not the same as the measured initiating particle distribution of 3.4 (2.7). This means that, although the predictions from the Modified Beremin and Bordet model are in general good, the calibration does not relate to measureable values so cannot easily be taken from experimental results or literature.

A significant advantage of the Developed Model compared with other LA cleavage models is that it offers a microstructure-property relation that remains as true to the physics as presently possible within the constraints of a homogenous, uncoupled material behaviour and the weakest-link assumption. The model directly takes microstructure data: the density of initiators and the probability distribution of their sizes, which can be determined by metallography. It requires the calibration of a single parameter; the rupture energy density scale, for which the additional information for positions of cleavage initiators can be determined by fractography. In the particular case reported here, the surface energy was also calibrated. It should be noted that the shape, scale and density are intrinsically related for a given material, so that the need to calibrate the surface energy could be eliminated for accurate experimental data. It has been shown, at least in principle, that by advancing the local failure probability expression and accounting for a "real" size distribution data, one can achieve improved predictions for cleavage fracture toughness, certainly in the lower transition region. The outcome at higher temperatures or low levels of constraint is not yet fully satisfactory, which is reflected in the shape of the predicted curve away from the calibration region, but the reason for this could be the third possibility described in the introduction: interactions between micro-cracks, the density of which increases with plasticity, may become strong enough to invalidate the weakest-link assumption behind the current model. This will be a subject of future investigations. One additional point worth noting is that the particle failure criterion in the proposed model is based on energy associated with failure normal to the maximum principal stress and on the assumption that the energy in the entire particle is lost when it ruptures; the latter introducing the particle size effect. Further investigation is necessary to support these assumptions or propose alternatives for all stress triaxialities and plastic strain levels. A micromechanical model of brittle particle in a plastically deformed matrix is used in an ongoing work to investigate these questions; the results will be reported in a future publication.

It is emphasized that the implementation of the Developed Model required only the changes of the tensile properties with temperature and irradiation when assessing the changes in the cleavage fracture toughness. Otherwise it uses two parameters (the position of the master curve was experimentally determined) calibrated at a single temperature for the high constraint, un-irradiated case. This indicates 
that the proposed model could be used predicatively, as it is here, without foreknowledge of the toughness values and without the need for further calibration.

\section{Conclusions}

The main conclusions from this paper are:

1) The proposed model is based on an improved criterion for individual probability of failure, i.e. probability of particle rapture and micro-crack propagation, and requires a simple set of experimental data for calibration: size distribution and density of the cleavage initiating particles from metallography and a limited range of positions of cleavage initiation sites from fractography.

2) The calibration can be performed at a single temperature for the model to be used predicatively over a range of temperatures, as well as a range of different irradiation and constraint conditions; model parameters remain constant, while the change of toughness arises only from the changing deformation properties, i.e. the material's stress-strain curve variation with temperature and/or irradiation.

3) The initiation sites predicted by the model, represented by the profile of the individual failure probabilities ahead of a macro-crack, correspond very well with the initiation sites profile for Material A determined experimentally over a range of temperatures. The correspondence between the two profiles provides evidence that the model links successfully the microstructure data, size distribution and density of particles, to longer-scale behaviour within the fracture process zone of the macro-crack.

4) The cleavage toughness predictions of the model are in good agreement with both experimental data and Master Curve fits in the un-irradiated high constraint state. It is also noted that the other approaches modelled here also provide good agreement for there conditions.

5) On changing the tensile properties in the finite element analyses to consider irradiation the spread of experimental results is well predicted for Material A. The predicted reference temperature shift is smaller than the experimentally determined, yet within acceptable limits.

6) When considering the effect of low constraint conditions against high constraint conditions the results show that the low constraint cases exhibit a more rapid DBT transition whilst still following the experimental data. The predicted reference temperature shift is again in good agreement with the experimentally determined shift, although the transition shape is different.

7) The model shows promising results for the combination of low-constraint and irradiation effects, which constitutes the majority of assessment needs in the engineering practice, although further works is required to improve the model's predictions at higher levels of fracture toughness under these condition, which is expected to be achieved by a better accounting for plasticity. The model is more sensitive to the effects of constraint than previous models; which is a further area of ongoing investigation. Given the good correlation of the calibration to measured data the sensitivity to constraint allows a useful framework to modify to better account for constraint.

\section{Acknowledgements}

The support from BNFL to Jivkov via endowment for Radwaste Research is gratefully acknowledged. James and Ford would also like to acknowledge assistance from colleagues at AMEC and to PERFORM60.

\section{References}

[1] A. Pineau, Development of the local approach to fracture over the past 25 years: theory and applications. Int J Fract 138 (2006) 139-166.

[2] F.M. Beremin, A local criterion for cleavage fracture of a nuclear pressure vessel steel. Metal Trans 14A (1983) 2277-2287.

[3] F. Mudry, A local approach to cleavage fracture. Nucl Eng Design 105 (1987) 65-76.

[4] F. Minami, A. Bruckner-Foit, D. Munz, B. Trolldenier, Estimation procedure for the Weibull parameters used in the local approach. Int J Fract 54 (1992) 197-210. 
[5] C. Ruggieri, R.H. Dodds Jr., A transferability model for brittle fracture including constraint and ductile tearing effects: a probabilistic approach. Int J Fract 79 (1996) 309-340.

[6] X. Gao, R.H. Dodds Jr., Constraint effects on the ductile-to-brittle transition temperature of ferritic steels: a Weibull stress model. Int J Fract 102 (2000) 43-69.

[7] J.P. Petti, R.H. Dodds Jr., Calibration of the Weibull stress scale parameter, $\sigma_{u}$, using the Master Curve. Eng Fract Mech 72 (2005) 91-120.

[8] B. Wasiluk, J.P. Petti, R.H. Dodds Jr., Temperature dependence of Weibull stress parameters: Studies using the Euro-material. Eng Fract Mech 73 (2006) 1046-1069.

[9] P. Akbarzadeh, S. Hadidi-Moud, A.M. Goudarzi, Global equations for Weibull parameters in a ductile-to-brittle transition regime. Nucl Eng Design 239 (2009) 1186-1192.

[10] Y. Cao, H. Hui, G. Wang, F.-Z. Xuan, Inferring the temperature dependence of Beremin cleavage model parameters from the Master Curve. Nucl Eng Design 241 (2011) 39-45.

[11] G. Xiaosheng, G. Zhang, T.S. Srivatsan, A probabilities model for prediction of cleavage fracture in the ductile-to-brittle transition region and the effect of temperature on model parameters. Mater Sci Eng 415A (2006) 264-272.

[12] M. Kroon, J. Faleskog, H. Oberg, A probabilistic model for cleavage fracture with a length scale Parameter estimation and predictions of growing crack experiments. Eng Fract Mech 75 (2008) 23982417.

[13] S.R. Bordet, A.D. Karstensen, D.M. Knowles, C.S. Wiesner, A new statistical local criterion for cleavage fracture of steel. Part I: model presentation. Eng Fract Mech 72 (2005) 435-452.

[14] J. Hohe, V. Hardenacke, S. Luckow, D. Siegele, An enhanced probabilistic model for cleavage fracture assessment accounting for local constraint effects. Eng Fract Mech 77 (2010) 3573-3591.

[15] A.P. Jivkov, D.P.G. Lidbury, P. James, Assessment of local approach methods for predicting endof-life toughness of RPV steels. In Proc PVP2011 (2011) paper 57546, Baltimore, USA.

[16] K. Wallin, T. Saario, K. Torronen, Statistical model for carbide induced brittle fracture in steel. Metals Sci 18 (1984) 13-16.

[17] L.C.A. Folch, F.M. Burdekin, Application of coupled brittle-ductile model to study correlation between Charpy energy and fracture toughness values. Eng Fract Mech 63 (1999) 57-80.

[18] G. Bernauer, W. Brocks, W. Schmitt, Modifications of the Beremin model for cleavage fracture in the transition region of a ferritic steel. Eng Fract Mech 64 (1999) 305-325.

[19] A. Pineau, Modeling ductile to brittle fracture transition in steels - micromechanical and physical challenges. Int J Fract 150 (2008) 129-156.

[20] B.Z. Margolin, V.A. Shvetsova, A.G. Gulenko, V.I. Kostylev, Prometey local approach to brittle fracture: Development and application. Eng Fract Mech 75 (2008) 3483-3498.

[21] Q.M. Li, Strain energy density failure criterion. Int J Solids Struct 38 (2001) 6997-7013.

[22] Private communication, PERFORM60 project

[23] ASTM E 1921-05, Standard Test Method for Determination of Reference Temperature T0 for Ferritic Steels in the Transition Range. ASTM, 2005.

[24] Private communication, S.R. Ortner, J. Duff, 2005.

[25] ABAQUS 6.11, DS Simulia Coorp, 2011.

[26] A. Hung, I. Yarovsky, J. Muscat, S. Russo, I. Snook, R.O. Watts, First-principles study of metallic iron interfaces, Surface Science 501: 261-269, 2002. 


\section{A Calibration}

The same approach for calibration was followed for each of the local approach models, which were calibrated to predict a 50 percent failure probability at $K=100.3 \mathrm{MPa} \sqrt{\mathrm{m}}$ at the experimentally determined $T_{0}$ temperature for the un-irradiated high constraint condition. All but one of the variables, typically those which described the particle distribution, were set to realistic physical values, with the remaining variable, which typically controlled critical crack size or particle failure, was then modified iteratively. Once calibrated for a material at the high constraint un-irradiated condition, the approach is not changed for subsequent changes in temperature, constraint or irradiation. Which variables were set and which was altered iteratively is shown in Table A1.

Table A1. Summary of the variables in each model and how they were used to calibrate the approaches.

\begin{tabular}{|l|c|c|}
\hline Local Approach Model & Set variables & Iteratively modified variable \\
\hline Modified Beremin & $k=1$ & $\sigma_{u}=2789.3$ \\
& $m=19$ & (hence $\sigma_{u} V_{0}{ }^{1 / m}=0.14738$ ) \\
& $V_{0}=0.001$ & $\sigma_{u}=2106.1$ \\
\hline Bordet & $V_{0}=0.001$ & \\
& $\sigma_{t h}=1000$ & \\
& $m=19$ & $\gamma_{s}+\gamma_{p}=0.005484$ \\
& $\gamma=6$ & \\
\hline WST & $\sigma_{0}=3000$ & $\gamma_{s}=0.002029$ \\
& $m=4$ & \\
\hline Developed Model & $\beta=2.7$ & \\
& $\psi_{0}=10000$ & \\
\hline
\end{tabular}

